ويزگ گیى هاى رشدى و ترموديناميكى فرانكنيا در بسترهاى كشت مختلف در ديوار سبز خارجى

\author{
منصوره جوزاى'، فاطمه كاظمى ب* و امير فتوت
}



جكيده















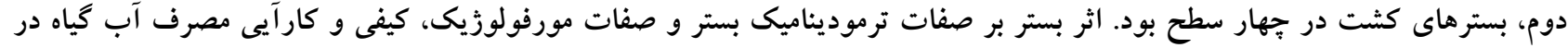













وازههاى كليدى: سيستم عمودى، توسعه بايدار، گياه يوششى، كارآيى مصرف آب، مواد بازيافتى



$$
\text { فردوسى مشها، ايران. }
$$








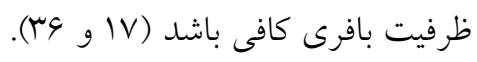









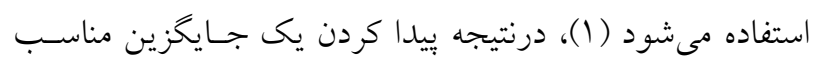



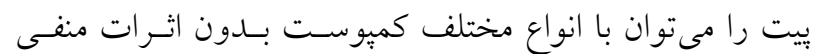


طرفى سالانه ميليونها تن ضايعات كشاورزى دامى و شهرى در






خاكبرى يوسيده و ورمى كميوست اشاره كرد (r) (1).











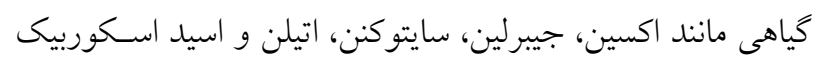


كاربرد كميوست قارج بهعنوان جايخزين ييت و كوكوييت توصسيه







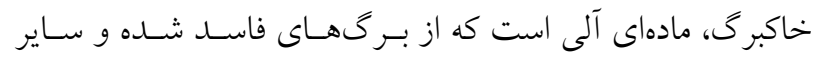

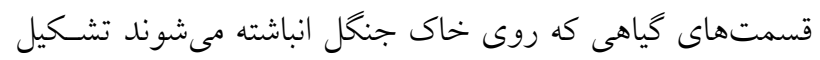

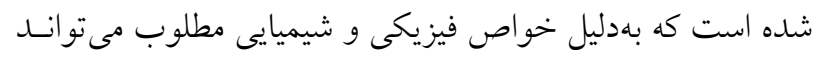
بهنوان بستر در رشد كياهجه استفاده شود (TV).





فضاهاى سـبز شـهرى قسـمتى از فضـاهاى بـاز هسـتند كـه در




امروزه بهدليل افزايش تراكم ساختوساز در كشورهاى در حسال












نماهاى سبز يكى از خزينههايى هستند كه با استفاده بهينـه از بـاز




عمودى كاركردهاى اكوسيستمى و زيستمحيطى بـر اى شهـرها





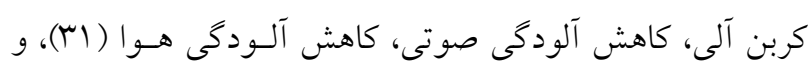

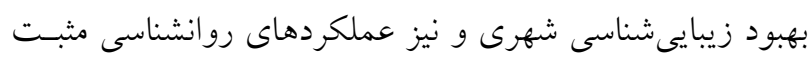



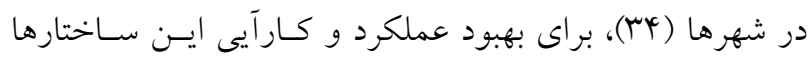



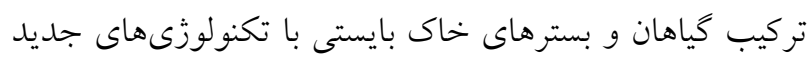



حداكثرى را براى اين سيستمها ايجاد كنند (11). تاكنون فناورىهاى زيادى براى ايجاد ديوارهاى سبز بـهـــار



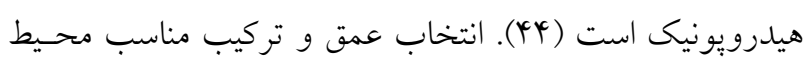
كشت در ساختارهاى عمودى سـبز اهميـت بسـز ايى دارد (YN).



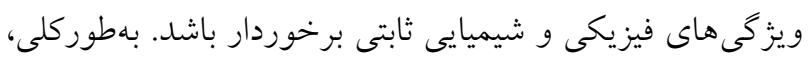






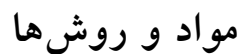
شرايط و مكان اجراى آزمايش اين تحقيق طى خرداد لو



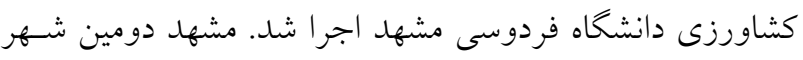






11 دقيقه شمالى، ه9 درجه و و4 درجه شرقى). براى انجام ايسن


عوامل ايجاد سايه و در جهـت رو بـه جنسوب اسـتقرار يافتنــ. سيستم كشت عمودى مورد استفاده در اين طرح روش موسـوم به سيستم كشت عمودى پاكتى بـود و ديوارهـاى سـبز احسـاث

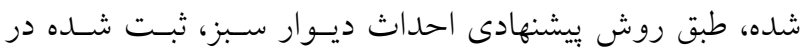















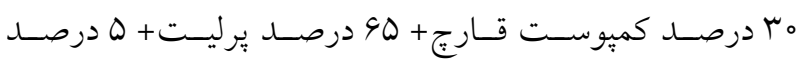
ورمى كمبوست و خاك معمـول مـورد اسـتفاده در فضـاى سـبز


دامى يوسيده به خاك لومى تشكيل مىشود)) و كياه مـورد نظـ فرانكنيا (Frankenia thymifolia) بود. سيستمب آبيـارى در ايسن




اساس دبى قطرهجيكانها اعمال شد.

صفات مورد بررسى - (1) - (1)

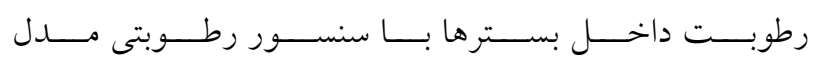

رشد سريع اما غيرتهاجمى اسـتفاده مسى شـود. در اكثــ مـوارد،


بهخوبى در خاكهاى كمعمق و خشكى رشد كنند، قابـل توجـهـ


ضمن افزايش ميزان سرانه فضاى سبز، در جهـار فصـل كياهـان جهره سبز داشته باشد (T) (Y).




براى جاهايى كه كاشت جّمن با مشكل مواجه است، بـهــار بـرد.

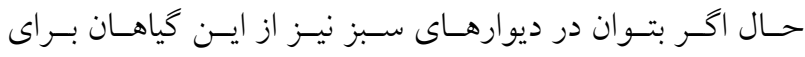

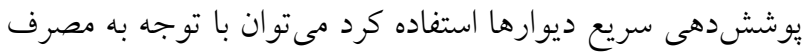


ديوارها را افزايش داد. جمن كوير يا فرانكنيا يكى از ايـن كياهـان


Frankeniaceae




ساقهاى آن رونده است كه بهدليل داشتن انعطاف در روى زمسين





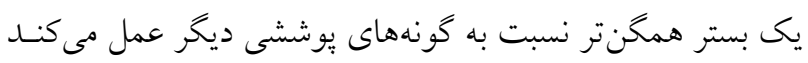



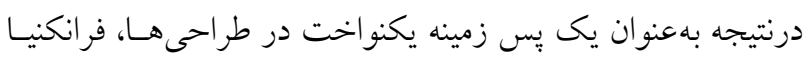













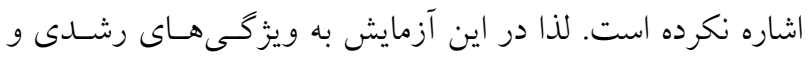
عملكردى كونه فرانكنيا در بسترهاى كشـت مختلـف در شـر ايط كشت در ديوارهاى سبز خارجى مى يردازيم. 
ميانخين دادهها با استفاده از آزمون تـوكى و رسـم نمودارهـا بـا



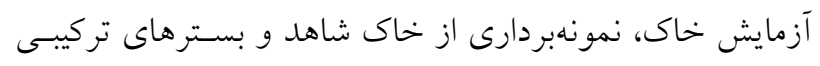





نتايج نتايج مربوط به تجزيه واريانس صـفات مـورد انـدازهيـــ درى جدول (r) تنظيم شده است. مطابق با جدول تجزيه واريانس اثرات ساده فصل و بستر كشت لتش انت






احتمال حداقل ينج درصد معنىدار شد.

\section{ارتفاع گياه}

مطابق با جدول (r)، اثرات ساده و متقابل فصل و بسـتر كشـت



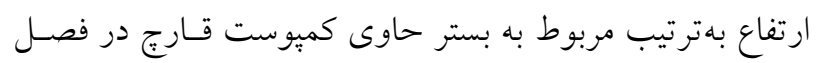
بهار و كمترين مربوط به بستر خاك شاهد در فصل زمستان بود.









اختلاف شاخص رشد نيز در بين بسترها و فصـول مختلـف از


فصل نيز در سطح احتمال ينج درصد اخستلاف معنسى دار نشـان دادند (جدول \&). در هر جهار بستر كشت در زمستان يك افت


رشد مربوط به بهار است. بستر كشت حساوى كميوسـت قـارج

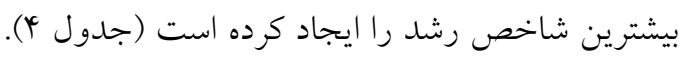

(USA, EXTECH MO750) در ساعت rا ظهر قبل از انجام آبيارى بهصورت هفتكى تعيسين



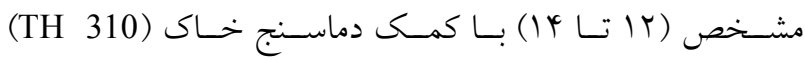


ميزان بوششدهـى گياهى با استفاده از كوآدرات ساخته شده بــهـ


ارتفاع كياهان با خطكش و شمارش تعداد كـره بـهروش شـمارش نيز بهصورت ماهانه صورت كرفت. درجه سـلامتى (ايـن شـاخص

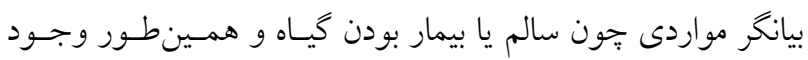





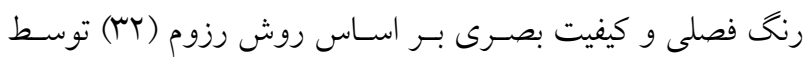











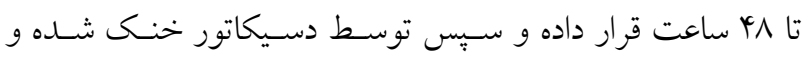


(Efficiency

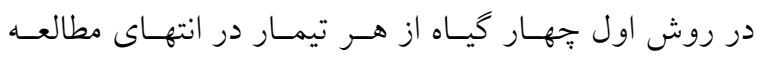






آب مصرفى دوره تيمار محاسبه شد (YY). كارآيى مصرف آب (g. L L $\left.{ }^{-1}\right)=$ ميزان آب مصرفى / (زيستتوده اوليه - زيستتوده نهايى) در روش دوم بــا تقسـيم تغييــر شـاخص رشـــ (اخـتلاف بـين










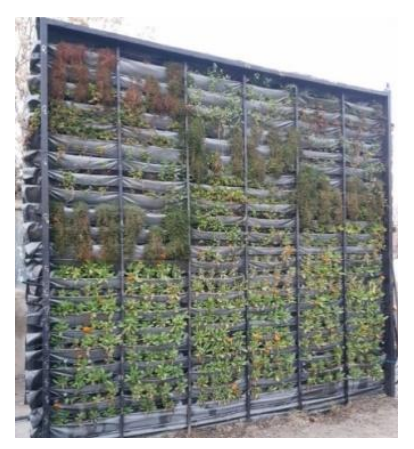

QV



91 بهار



9V تابستان



QV زمستان

شكل 1. نماى كلى طرح آزمايشى در جهار فصل سال (بخش فوقانى ديوارهاى سبز مرتبط با ئزوهش گزارش شده در اين مقاله است.)

تيرهترين رنغ مربوط به كياهان در بستر كشت كميوست قـارج

$$
\text { درصد بوشش (جدول أ). }
$$

نتايج تجزيه واريانس براى درصد يوششدهى كياه نشان مىدهـد كه اثرات ساده و متقابل فصل و بسـتر كشـت اخـتلاف معنسىدار

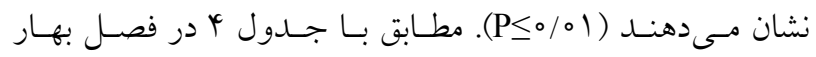
بيشترين بوششدهى مشهود است درحالى كه در زمستان بهعلـت بهخواب رفتن كياه و خشك شدن و ريزش شاخساره، افت شديد درصد يوششدهى در تمامى بسـترها رؤيست شـــ. در هـر حسال بيشترين و كمترين درصد بوششدهى اين گياه در شـرايط ديسوار سبز بهترتيب مربوط به بستر كميوست قارج و خاك بود.


صفت درجه سلامتى در گياه فرانكنيا علاوه بر اثرات ساده فصل و بستر كشت، اثـر متقابـل در سـطح احتمـال يـك درصــ نيـز معنى دار بود (جــدول ب). در تمـامى بسـترها در فصـل يـاييز و
كيفيت بصرى صفت كيفيت بصرى بهصورت معنى دارى تحست تـأثير فصـل و


متقابل نيز معنى دار بود. در تمامى فصـول بسـتر كشـت حساوى كميوست قارج نسبت به سه بستر ديخر توانست كيفيت بصـرى


تمامى بسترها افت شديد كيفيت بصرى را نشان دادند بهطـورى كه كمترين كيفيت مربوط به بستر كشت خاك در زمسـتان بـود



\section{رنخ فصلى}

همانطور كه نتايج جدول ب نشان مىدهــ در ارتبـاط بـا رنـع فصلى اثرات ساده و اثر متقابـل فصـل و بسـتر كشـت اخـتلاف معنى دارى با يكديخر دارند ( 1 (P>0/0). فرانكنيا در فصل تابستان علاوه بر فصل بهار بهترين كيفيت رنخ را ايجاد كرده و در دو فصل سرد سال كيفيت رنخ كـاهش يافتـه اسـت. بسـتر كشـت




جدول ا. خصوصيات شيميايى بسترهاى كشت مورد استفاده در آزمايش

\begin{tabular}{|c|c|c|c|c|c|c|c|c|c|}
\hline \multirow{2}{*}{$\mathrm{pH}$} & $\mathrm{EC}$ & \multirow{2}{*}{$\mathrm{C} / \mathrm{N}$} & $\mathrm{OM}$ & $\mathrm{OC}$ & $\mathrm{Mg}$ & $\mathrm{K}$ & $\mathrm{P}$ & $\mathrm{N}$ & \multirow[b]{2}{*}{ بستر } \\
\hline & $(\mathrm{dS} / \mathrm{m})$ & & \multicolumn{6}{|c|}{ (درصد) } & \\
\hline G/AV & $1 / \pi T$ & $r \circ / \varphi$ & $11 / \mu$ & $9 / 09$ & $\circ / N r$ & $\circ / \mathcal{C}_{0}$ & $\circ / \circ \Delta$ & $0 / M T$ & 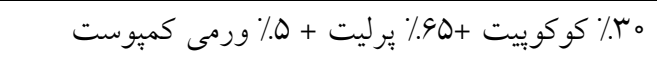 \\
\hline$V / T I$ & $1 / \pi q$ & $V / \circ 9$ & $10 / \mu$ & $1 / 19$ & $\circ / \mathrm{V}$ & سח/ם & $\circ / \wedge$ & $1 / T \Delta$ & 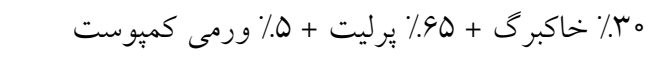 \\
\hline$V / 19$ & T/A & $10 / 1$ & $1 / / 1$ & $10 / 9$ & $1 / \pi V$ & $\circ / Q V$ & $0 / 09$ & $\circ / \mathrm{V}$ &  \\
\hline$N / \circ$ & $1 / T r$ & $r \circ / 1$ & $r / \Delta T$ & T/AT & $0 / T G$ & $0 / 01$ & \%/०० &.$/ 14$ & خاى معمول مورد استفاده در فضاى سبز بهعنوان شاهد \\
\hline
\end{tabular}



جدول r. خصوصيات فيزيكى بسترهاى كثت مورد استفاده در آزمايش

\begin{tabular}{|c|c|c|c|c|}
\hline وزن مخصوص & تخلخل & نقطه يزّمرگى دائم & ظرفيت نخهارى آب & بستر \\
\hline$\left(\mathrm{g} / \mathrm{cm}^{3}\right)$ & & (درصد) & & \\
\hline $0 / 49$ & VA & $1 / \circ 0$ & $v v^{4}$ & 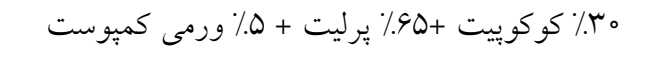 \\
\hline $0 / 90$ & pr & N/৭9 & $99 / 1$ & 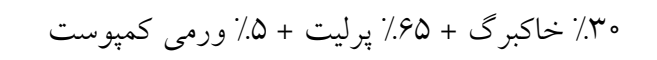 \\
\hline $0 / \pi 9$ & $\Delta G$ & $4 / 09$ & $11 r$ & 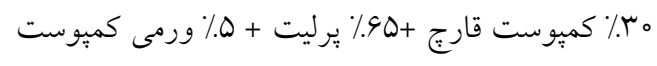 \\
\hline$\circ / N Q$ & $\wedge$ & Y/NY & $\mu_{\Lambda}$ & خاك معمول مورد استفاده در فضاى سبز بهعنوان شاهد \\
\hline
\end{tabular}

بيشترين دماى بسـتردر تابسـتان ثبـت شـده اسـت كـهـ احتمـالاً بهعلت بوششدهى سطح خاك يايين اين گياه در تابسـتان بـوده

مقايسه ميانكين هاى بسترهاى مختلف در كونه فرانكنيا نشان مىدهد بيشترين و كمترين رطوبت بستر مربوط به كوكوييـت و كميوست قارج بود. در كونه فرانكنيـا فصـل تابسـتان بيشـترين حفظ رطوبت بستر را دارد در حالى كـه ديخـــ فصـلهـا از نظـر آمارى يكسان عمل كردند (جدول ه).

كارآيى مصرف آب بر اساس زيست توده كل در مورد اين صفت مطابق جدول (r) در كونـه فرانكنيـا اثـرات ساده فصل و بستر كشت در سطح احتمال حــاقل يـك درصــ


مصرف آب مربوط بـه كميوسـت قـارج و خـاكبرى و در بـين فصول نيز، بيشترين كارآيى مصرف آب مربوط به يـاييز و بهـار



زمستان كاهش درجه سلامتى را مشاهده كرديم. اما اين كـاهش در دو بستر كميوست قارج و خاكبرگ كمتر بود (جدول \&).


مطابق با جدول تجزيه واريـانس (جــول r) اثـرات سـاده بسـتر كشت و فصل در شاخص تعداد گره اختلاف معنى دار نشان دادند


نشان داد كه بيشترين تعداد گره مربوط به بستر حساوى كمبوسـت قارج و كمترين تعداد گره مربوط به بستر خاك بود. در فصل بهار بيشترين تعداد گره مشهود است اما كمترين گره در گونه فرانكنيـا در زمستان ثبت شده است (جدول ه).

\section{دما و رطوبت بستر}

مطابق جدول تجزيه واريانس (r) در كياه فرانكنيـا تنهـا اتـرات ساده فصل و بستر كشت دما و رطوبت بستر در سـطح احتمـال يك درصد اختلاف معنىدار نشان مسىدهـــ. در گونـه فرانكنيـا 





جدول fا. مقايسه ميانخينهاى مربوط به اثرات متقابل فصل و بستر كشت بر برخى صفات رشدى و كيفى گياه فرانكنيا

\begin{tabular}{|c|c|c|c|c|c|c|c|}
\hline درجه سلامتى & يوششدهى & رنخ فصلى & كيفيت بصرى & شاخص رشد & $\begin{array}{l}\text { ارتفاع } \\
\text { (cm) }\end{array}$ & & \\
\hline$\varphi / 9 \Lambda^{a}$ & $r \circ / Q^{1}$ & $\omega^{\mathrm{a}}$ & T/YYhi & rysl def & $T Y / q^{\mathrm{efg}}$ & بستر & \multirow{4}{*}{ تابستان } \\
\hline$\Delta^{\mathrm{a}}$ & $r \mu / M^{c k}$ & $\Delta^{\mathrm{a}}$ & $r / \mathcal{Y}_{\circ} \mathrm{gh}$ & ryqudef & $r \& / q^{c d e}$ & بستر ب & \\
\hline$\Delta^{\mathrm{a}}$ & $r q / q^{i}$ & $\Delta^{\mathrm{a}}$ & $r / 9 \Delta^{g}$ & rAVGcd & $\mu_{\circ} / l^{b}$ & بستر r & \\
\hline$Y / \Delta \Delta^{c d}$ & $1 \mathrm{r} / \mathrm{A}^{\mathrm{m}}$ & $\varphi / 9 \Delta^{b c d}$ & $1 / V Y^{j}$ & $\Gamma \circ \Delta q^{\mathrm{fgh}}$ & $Y \Psi / q^{\text {efgh }}$ & بستر ب ب & \\
\hline$r / N \mu^{f}$ & $\varphi \circ / \mu^{g}$ & $\varphi / 1 q^{e f}$ & F/AYC & $q^{\text {efg }}$ & $r \mu / /_{\text {fghi }}$ & بستر & \multirow{4}{*}{ ياييز } \\
\hline $4 / 91^{\mathrm{c}}$ & $\Delta \mathrm{Or} / \mathrm{Q}^{\mathrm{e}}$ & $\varphi / \mu \varphi \operatorname{def}$ & $r / 90^{\circ}$ & $r r q \wedge^{\text {defg }}$ & $r / /$ ghi & بستر r & \\
\hline$y / q y^{a b}$ & $90 / 4 b$ & $Y / V q a b c$ & $\varphi / q \mu^{\mathrm{ab}}$ & MAYycde & $r V / \wedge^{b c d}$ & بستر r & \\
\hline$r / \mu q g$ & $\mu \mathrm{c} / \mathrm{N}^{\mathrm{h}}$ & $r / \Lambda q^{h}$ & $r / \circ \varphi^{f}$ & rq19Ghi & $r \circ / q^{\mathrm{ij}}$ & بستر ب & \\
\hline$r / \mu T^{g}$ & $r G / y^{c h}$ & $r / 09 g$ & $r / 99^{e}$ & $Y \Delta \circ \wedge^{\mathrm{ij}}$ & $Y I / T^{h i}$ & بستر 1 & \multirow{4}{*}{ زمستان } \\
\hline$y / \mu$ de & $r Q / \mathbb{F}^{\mathrm{ff}}$ & $\varphi / 0 \phi^{f}$ & $\varphi / / \mu^{\mathrm{d}}$ & $r \vee \sim \Lambda^{h i}$ & rrghi & بستر r & \\
\hline$y / q^{b b c}$ & $91 / 1^{\mathrm{c}}$ & $\varphi / 9 \Delta^{b c d}$ & $\varphi / \mu \mu^{c}$ & MYq. def & $r \varepsilon / /$ def & بستر r & \\
\hline$r / \Delta \Delta^{h}$ & $r G / \mathcal{G}^{\mathrm{j}}$ & $r / 19^{i}$ & $r / l \circ \mathrm{i}$ & $r / \wedge \Delta^{j}$ & $\mathrm{IV} / \mathrm{V}^{\mathrm{j}}$ & بستر ب & \\
\hline$\varphi / \circ \varphi^{e}$ & $q V^{f}$ & $Y / 0$ ०cde & $y / 4 \varphi_{c}$ & $4 \backslash \wedge q^{b c}$ & $\left\lceil\Lambda / Y^{b c d}\right.$ & بستر 1 & \multirow{4}{*}{ بهار } \\
\hline$\varphi / \Delta \wedge^{c d}$ & $\Delta V / T^{d}$ & $\varphi / \wedge \varphi^{a b}$ & $\varphi / 9 \wedge^{\mathrm{bc}}$ & FOrA ${ }^{b}$ & $r q / r^{b c}$ & بستر r & \\
\hline$\varphi / 9 y^{\mathrm{ab}}$ & $\vee \wedge / \wedge^{\mathrm{a}}$ & $\varphi / 9 r^{a b}$ & $\varphi / 9 \varphi^{a}$ & $01 \circ \Delta^{a}$ & $r q / l^{a}$ & بستر ب & \\
\hline$r / \varphi \circ g$ & $\mu \mathrm{r} / \mathrm{gh}$ & $r / l{ }^{c h}$ & $r / q Y^{f f}$ & $\mu_{0} \& r^{\mathrm{fgh}}$ & $r Y / /^{h i j}$ & بستر ب & \\
\hline
\end{tabular}

حروف لاتين كوجى متفاوت در هر ستون جدول، نشاندهنده اختلاف معنادار بر اساس آزمون توكى در سطح احتمال حداقل ينج درصد است.

آب را نسب به بسـتر خـاك در تمـامى فصـول افـزايش دادنــ بحث توسعه فناورى ديوارهاى سبز در سطح جهان در مسير بيشـرفت (جدول (ه) ( ) بوده و هر روز يروزههاى نوينى در اين زمينسه توسـط طراحسان


كار آيى مصرف آب بر اساس شاخص رشد ماند كارى اين ديوارها اسـت و ايـن جـز بــا داشـتن يـك بسـتر مطابق جدول (r) تنها اثرات ساده فصل و بستر كشت در سـطح بـ مناسب براى استقرار كياهان در آن فراهم نمىشـود. نتـايج ايسن احتمـال يـك درصــ در مـورد كـارآيى مصـرف آب اخـتلاف آزمايش نشان داد كه در بستر تركيبى حساوى كميوسـت قـارج، معنى دار نشان مىدهد. بيشترين كارآيى مصـرف آب بـر اسـاس كياه فرانكنيا ارتفاع بهترى داشت و روند رشدى آن نشان داد كه شاخص رشد مربوط به فصل تابستان و همجنين بستر كميوست اين كياه در فصل تابستان بعد از كشت سريع به استقرار رسيد و قارج و كمترين آن مربوط به خاك شاهد و فصـل زمسـتان بـود




جدول ه. مقايسه ميانخينهاى مربوط به اثرات ساده فصل و بستر كشت بر برخى ويزّكىهاى ترموديناميكى بستر و عملكردى گياه فرانكنيا



\begin{tabular}{|c|c|c|c|c|c|}
\hline $01009^{b}$ & $r / q^{b}$ & $r \circ / l^{b}$ & $r \Leftrightarrow / \mu^{\mathrm{a}}$ & $19 / 1^{\mathrm{c}}$ &  \\
\hline $0 / 0 r^{a}$ & $\Gamma / \Lambda^{b}$ & $Y Y / K^{c a}$ & $\mid y / Y^{b}$ & $r / / /^{b}$ &  \\
\hline $0 / 0 r^{a}$ & $\mu / \mu^{a}$ & $T Y / T)^{b}$ & $I K / N^{c}$ & $r y / /^{a}$ &  \\
\hline $0 / 0 \circ \gamma^{c}$ & $T / /^{c}$ & $19 / 0^{c}$ & $19 / \mathrm{V}^{\mathrm{ab}}$ & $19 / \mu^{\mathrm{d}}$ & خاك معمول مورد استفاده در فضاى سبز بهعنوان شاهد \\
\hline$\circ / \circ \circ V^{c}$ & $\varphi / \mu^{a}$ & $r q / 1^{a}$ & $r Y / \circ \varphi^{a}$ & $r T / T^{\mathrm{a}}$ & تابستان \\
\hline $0 / 01^{a}$ & $r / \Lambda^{b}$ & $19 / \mathrm{N}^{\mathrm{c}}$ & $1 N / Y^{b}$ & $r_{0} / \mathrm{c}^{\mathrm{b}}$ & ياييز \\
\hline $0 / 01^{b}$ & $1 / 9^{\mathrm{d}}$ & $1 / / Y^{b}$ & $19 / \mathrm{rb}^{\mathrm{b}}$ & $14 / q^{c}$ & زمستان \\
\hline$\% / r^{a b}$ & $r /\left.0\right|^{c}$ & $r_{0} / /^{b}$ & $\mid k / 4 b$ & $r \mu / \Lambda^{a}$ & بهار \\
\hline
\end{tabular}

حروف لاتين كوجك متفاوت در هر ستون جدول، نشاندهنده اختلاف معنادار بر اساس آزمون توكى در سطح احتمال حداقل ينج درصد است.









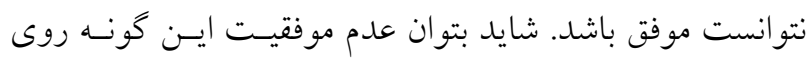
ظرفيت نخهلدارى عناصر غذايى و افزايش هورمسونهـاى تنظيم










نيتروزن، رشد كياه و ارتفاع افزايش مى بيابد.



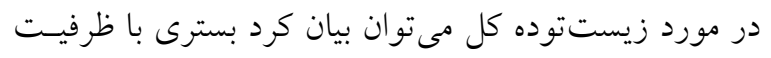




كمبوست بر افزايش ارتفاع با نتايج بهدست آمده روى بادمجـان،






كارآيى مصرف آب حتماً به معنى بهبود رشد يـا محصـول كلى

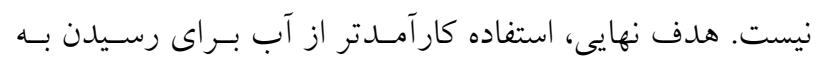

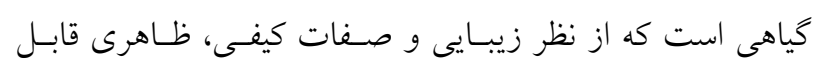








مصرف آب شدهاند (9).





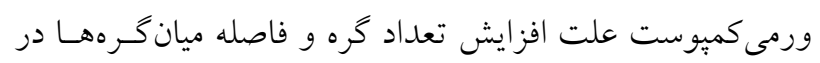









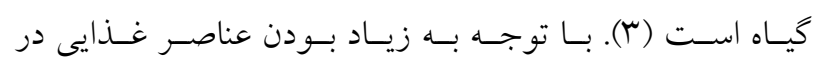

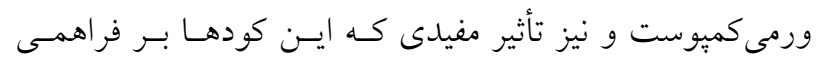



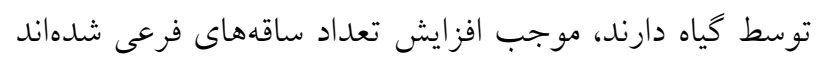

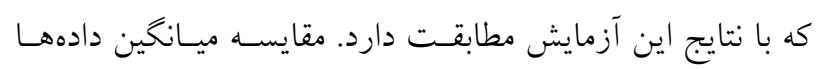

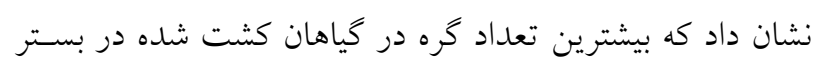

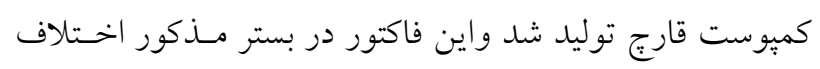

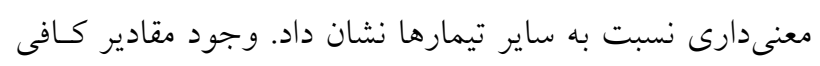














كميوست قارج خوراكى به خاك را در افزايش معنسى دار ارتفـاع سويا مؤثر دانستهاند (1) (l).






خود سبب حفظ سطح فتوسنتز كننده بمامدت طولانى مى شــود و






بسترهاى كشت تركيبى كمبوست قارج در درجه اول و در ساير



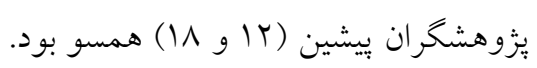















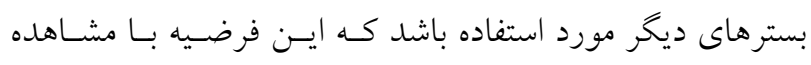


اثبات مىشود. بلوم (9) ييشنهاد كرد كه كار آيى مصـرف آب بـاتلاتر، بيشـتر درنتيجه كاهش مصرف آب بهدليل كـاهش تعـرق توسط كيـاه



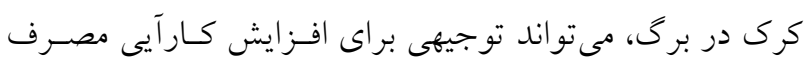









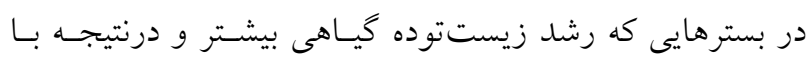

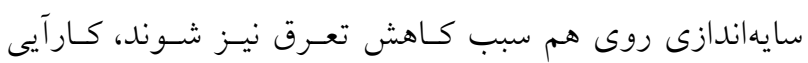



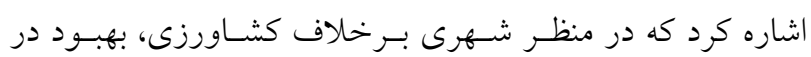


خاك بود و اين رويداد در فصل زمستان بهخاطر گرم نخهداشتن

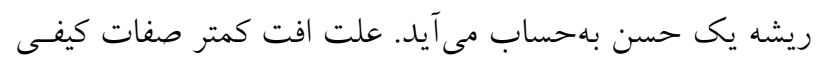

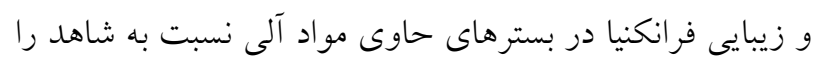

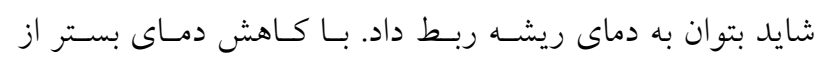

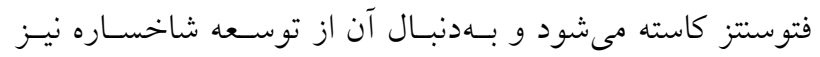

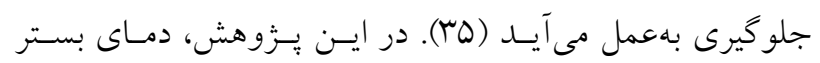

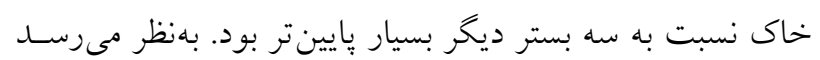

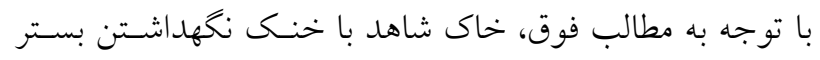
موجب كاهش جذب آب توسط كيـاه شـده و بنـابر اين ميـزان

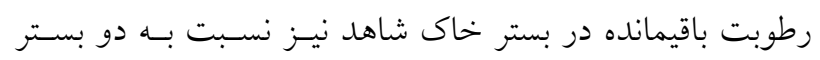

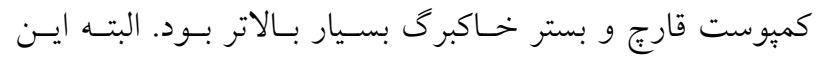
احتمال نيز وجود دارد كه وجود رطوبت بيشستر در بسـتر خـاك

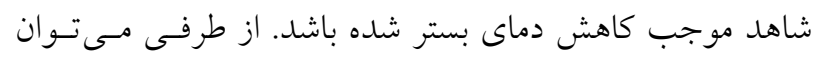





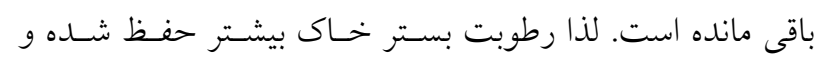

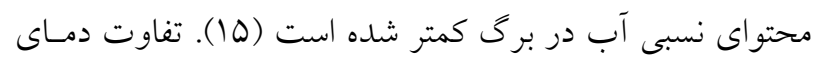

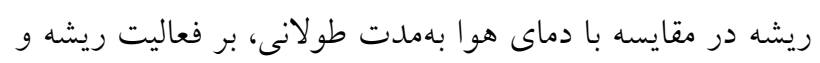

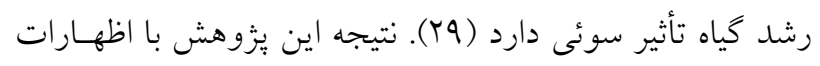

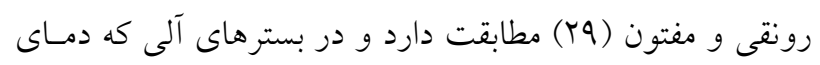

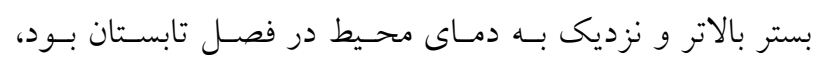

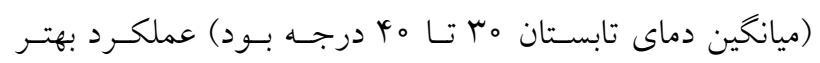

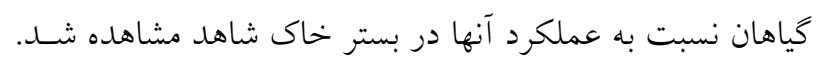

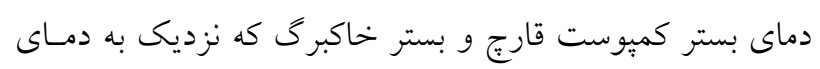

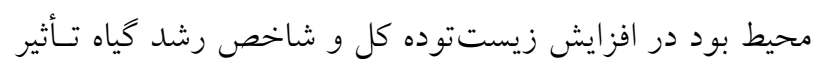
بسز بـ داشت.

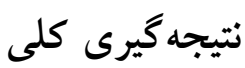

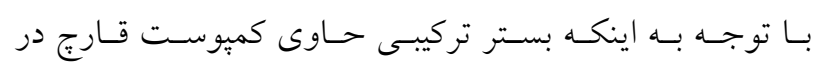

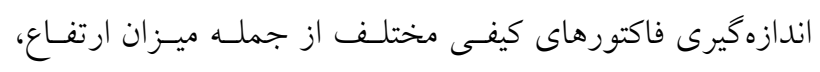

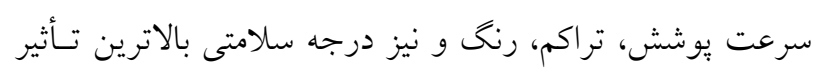

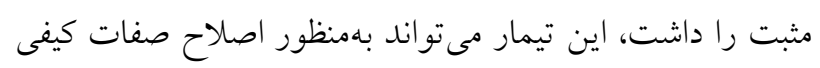

درجـه سـلامت، رنــ فصسلى و كيفيـت بصـرى فرانكنيـا در

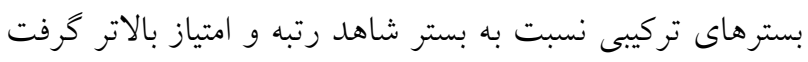

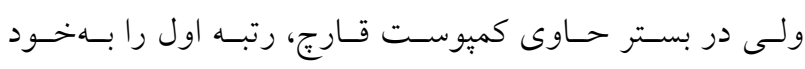

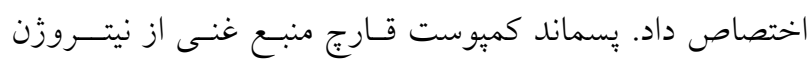

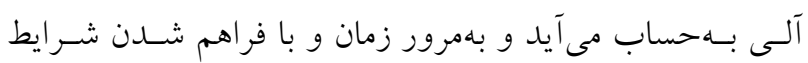

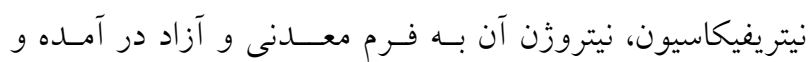

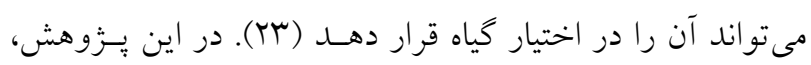
در اثر مصرف كميوست قـارج ميزان نيتروزن در خـاك و گيـاه

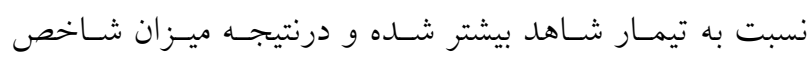

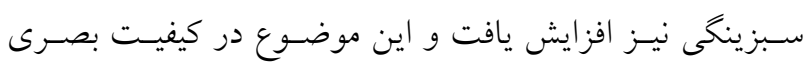
كَاه، درجه سلامت و رنح فصلى موثر است. اين يافته با نتسايج



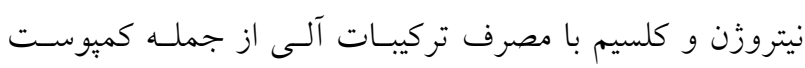


كَاهان افزايش مى ويابد.



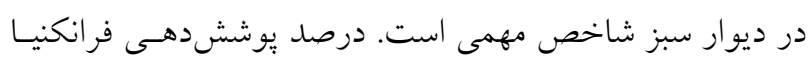

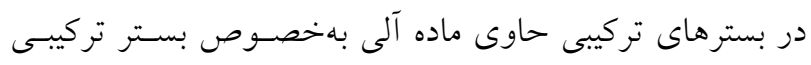

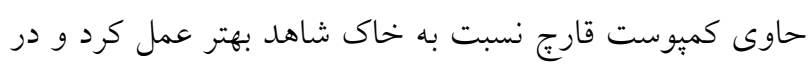
فصل بهار و تابستان نسبت به فصل وباييز و زمستستان در كياهـان

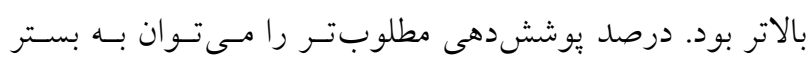

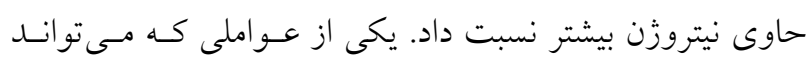

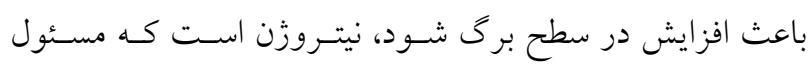

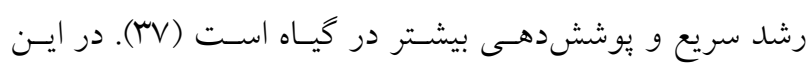

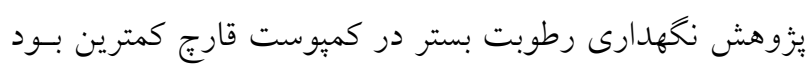

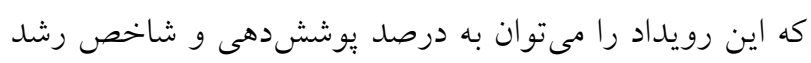

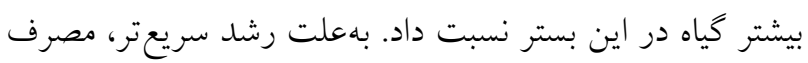

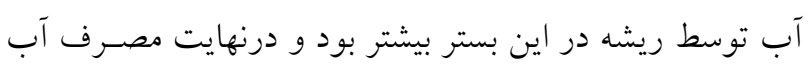

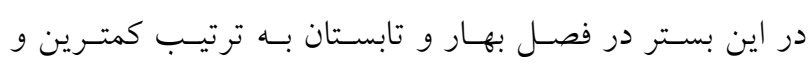
بيشترين بود.

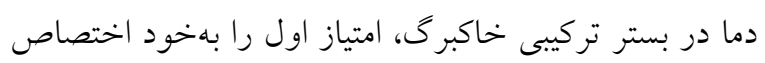
داد. بهطور كلى دما در بسترهاى حاوى مواد آلى بيشتر از بسـتر 


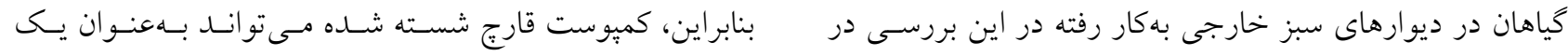

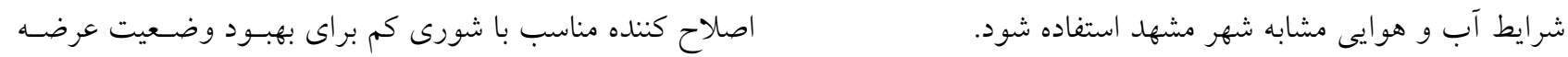

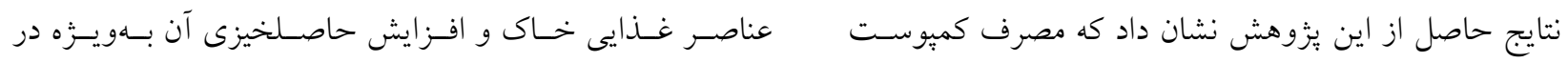

$$
\begin{aligned}
& \text { قارج خور اكى باعث افزايش ماده آلى و غلظـت عناصـر غـذايى اكهاى مناطق خشى و نيمهخشى استفاده شود. } \\
& \text { ضرورى برمصرف در خـاك در مقايسـه بـا تيمـار شـاهد شـــ }
\end{aligned}
$$

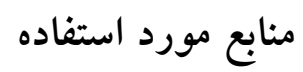

1. Abad, M., P. Noguera and S. Burés. 2001. National inventory of organic wastes for use as growing media for ornamental potted plant production: case study in Spain. Bioresource Technology 77: 197-200.

2. Arancon, N. Q., C. A. Edwards, R. M. Atieyh and J. D. Metzger. 2004. Effect of composts produced from food waste on the growth and yields of greenhouse peppers. Brioresource Technology 93: 139-143.

3 Atiyeh, R. M., S. Subler, C. A. Edwards, G. Bachman, J. D. Metzger and W. Shuster. 2000. Effects of vermicomposts and composts on plant growth in horticultural container media and soil. Pedobiologia 44: 579-590.

5. Barrett, G. E., P. D. Alexander, J. S. Robinson and N. C. Bragg. 2016. Achieving environmentally sustainable growing media for soilless plant cultivation systems- A review. Scientia Horticulturae 212: 220-234.

6. Blum, A. 2005. Drought resistance, water-use efficiency, and yield potential- are they compatible, dissonant, or mutually exclusive? Australian Journal of Agricultural Research 56(11): 1159-1168.

7. Caprotti, F. and J. Romanowicz. 2013. Thermal eco-cities: Green building and urban thermal metabolism. Journal of Urban and Regional Research 37(6): 1949-1967.

8. Elhami, H. 2015. Effects of drought stress on yield and quantitative characteristics and characteristics of four covered plant species with potential for use in Mashhad green space. Master thesis. Faculty of agriculture, Ferdowsi University of Mashhad.

9. Farooq, M., A. Wahid, N. Kobayashi, D. Fujita and S. M. A. Basra. 2009. Plant drought stress: effects, mechanisms and management. Agronomy for Sustainable Development 29: 185-212.

10. Fernandez-Canero, R., J. Ordovas and M. A. H. Machuca. 2011. Domestic gardens as water-wise landscapes: A case study in southwestern Europe. Hort Technology 21: 616-623.

11. Ghaffarian Hoseini, A. and N. Makaremi. 2012. The Concept of zero energy intelligent buildings (ZEIB): A review of sustainable development for future cities. British Journal of Environment and Climate Change 2(4): 339-367.

12. Gonani, Z., H. Riahy and K. Sharifi. 2011. Impact of using leached spent mushroom compost as a partial growing media for horticultural plants. Journal of Plant Nutrition 34: 337-344.

13. Hernandez-Apaolaza, L., A. M. Gasco, J. M. Gasco and F. Guerrero. 2005. Ruse of waste materials as growing media for ornamental plants. Bioresource Technology 96: 125-131.

14. Jonathan, S., O. Oyetunji, O. Olawuyi and P. Uwukhor. 2013. Application of Pleurotus ostreatus SMC as soil conditioner for the growth of soybean (Glycine max). Academia Arena 5: 54-61.

15. Jozay, M. and F. Kazemi. 2019. Evaluating the environmental performance of the growing media in a green wall system in a dry climate region. Desert 20: 217-230.

16. Kazemi, F., S. Beecham and J. Gibbs. 2011. Streetscape biodiversity and the role of bioretention swales in an Australian urban environment. Landscape and Urban Planning 101: 139-148.

17. Kazemi, F. and R. Mohorko. 2017. Review on the roles and effects of growing media on plant performance in green roofs in world climates. Urban Forestry \& Urban Greening 23: 13-26.

18. Keever, G. J., G. S. Cobb and R. McDaniel. 1986. Effects of container size, root pruning, and fertilization on growth of seedling pecans. Journal of Environmental Horticulture 4: 11-13.

19. Koch, K., R. Samson and D. Siegfried. 2019. Aerodynamic characterization of green wall vegetation based on plant morphology. Anexperimental and Computational Fluid Dynamics Approach 178: 34-51.

20. Kubilay Onal, M. and B. Topcuoglu. 2007. The effect of spent mushroom compost on the dry matter mineral content of pipper (Piper Nigrum) grown in greenhouse. Available online at: https://www.semanticscholar.org/paper/. Accessed 8 August 2019.

21. Lazzarin, R. M., F. Castellotti and F. Busato. 2005. Experimental measurements and numerical modelling of a green roof. Energy Building 37: 1260-1267.

22. Liuc, J. and B. Pank. 2005. Effect of compost and fertility levels on growth and oil yield of Roman chamomile. Scientia Pharmaceutica 46: 63-69. 
23. Medina, E., C. Paredets, M .A. Bustamante, R. Moral and J. Moreno-Caselles. 2012. Relationships between soil physico-chemical and biological properties in a soil amended with spent mushroom substrate. Geoderma 152-161.

24. Medrano, H., M. Tomás, S. Martorell, J. Flexas, E. Hernández, J. Rosselló, A. Pou, J. M. Escalona and J. Bota. 2015. From leaf to whole-plant water use efficiency (WUE) in complex canopies: Limitations of leaf WUE as a selection target. The Crop Journal 3: 220-228.

25. Qasemi Ghahsareh, M. and M. Kafi. 2009. Floriculture (Scientific and Practical Points), Volume 2. Moallef Publications.

26. Rabbani Kheir khah, S. M. and F. Kazemi. 2015. Investigating strategies for optimum water usage in green spaces covered with lawn. Desert 20: 217-230.

27. Rahimic, A., V. Komlen, D. Vukotic, N. Tanovic and J. Aliman. 2013. Utjecaj listinca na morfoloska svojstva pres a dnica bosiljka (Ocimum basilicum). 48th Croatian \& 8th International Symposium on Agriculture Dub rovnik, Croatia.

28. Razzaghmanesh, M., S. Beecham and F. Kazemi. 2014. The growth and survival of plants in urban green roofs in a dry climate. Science of the Total Environment 476-477: 288-297.

29. Ronaghi, A. and M. Maftoon. 2003. Hydroponics, A Practical Guide to Soilless Culture Practitioners, Shiraz University Press (In Farsi).

30. Rout, G. R. and S. Sahoo. 2015. Role of iron in planth growth and metabolisam. Agricultural Science 3: 1-24. doi: 10.7831/ras.3.1.

31. Romanova, A., K. Horoshenkov and A. Hurrell. 2019. An application of a parametric transducer to measure acoustic absorption of a living green wall. Applied Acoustics 145: 89-97.

32. Rozum, J. 2014. Irrigation effects on growth and visual quality of three ornamental grass species. Theses and Dissertations - Department of Horticulture \& Landscape Architecture. Colorado State University, Colorado, USA.

33. Run-Hua, Z., D. Zeng-Qiang and L. Zhi-Guo. 2012. Use of spent mushroom substrate as growing media for tomato and cucumber seedlings. Pedosphere 22: 333-342.

34. Sheweka, S. and N. Magdy. 2011. The living walls as an approach for a healthy urban environment. Energy Procedia 6: 592-599.

35. Shibani Rad, A., M. Haghighi and A. Honestly. 2014. Effect of temperature reduction and nitrogen reduction on physiological characteristics (growth and photosynthesis) of lettuce (Lactuca sativus). Process and Plant Function 4 (13): 121-132. (In Farsi).

36. Shi, Z. Q., F. Jobin-Lawler, A. Gosselin, G. Turcotte, A. P. Papadopoulos and M. Dorais. 2002. Effect of different EC management on yield, quality and nutraceutical properties of tomato grown under supplemental lighting. Acta Horticulturae 580: 241-247.

37. Soltani, A. 2009. Mathematical Modeling in Field Crops. JDM Press, Mashhad.

38. Soumare, M., F. Tack and M. Verloo. 2003. Effects of a municipal solid waste compost and mineral fertilization on plant growth in two tropical agricultural soils of Mali. Bioresource Technology 86: 15-20.

39. Tomati, U., A. Grappelli and E. Gali. 1987. The harmonic-like effect of earthworm casts on plant growth. Biology and Fertility of Soil 5: 228-294.

40. Stabler, L. B. and C. A. Martin. 2000. Irrigation regimens differentially affect growth and water use efficiency of two southwest landscape plants. Journal of Environmental Horticulture 18(2): 66-70.

41. Tzortzakis, N., S. Gouma, C. Paterakis and T. Manios. 2012. Deployment of municipal solid wastes as a substitute growing medium component in marigold and basil seedlings production. The Scientific World Journal 2012: 1-6. doi: $10.1100 / 2012 / 285874$.

42. Uzun, I. 2004. Use of spent mushroom compost in sustainable fruit production. Journal of Fruit and Ornamental Plant Research 12: 157.

43. Vahdati, N., A. Tehranifar and F. Kazemi, 2017. Assessing chilling and drought tolerance of different plant genera on extensive green roofs in an arid climate region in Iran. Journal of Environmental Management 192: 215-223.

44. Weinmaster, M. 2009. Are green walls as "Green" as they look? An introduction to the various technologies and ecological benefits of green walls. Journal of Green Building 4: 3-18.

45. Zhang, X. 2013. Going green: initiatives and technologies in Shanghai World Expo. Renewable and Sustainable Energy Reviews 25: 78-88. 


\title{
Evaluation of the Growth and Thermodynamic Properties of Frankenia on Different Growing Media in an External Green Wall
}

\author{
M. Jozay ${ }^{1}$, F. Kazemi ${ }^{*}$ and A. Fotovat ${ }^{3}$
}

(Received: January 25-2020; Accepted: June 22-2020)

\begin{abstract}
Currently, the idea of integrating nature and buildings through green roofs and green walls has been considered as a sustainable strategy in cities. However, studies on the substrates which allow the plants to fully establish in these systems are still lacking. It is also a challenge to find the substrates which provide the plants with proper growth, nutrition, and environmental sustainability. This study aimed to evaluate the performance of Frankenia thymifolia in four types of growing media $(30 \%$ cocopit $+65 \%$ perlite $+5 \%$ vermicompost, $30 \%$ soil $+65 \%$ perlite $+5 \%$ vermicompost, $30 \%$ mushroom compost $+65 \%$ perlite $+5 \%$ vermicompost and common soil used in the green spaces as the control) in growing conditions of the external green walls. The experiment was conducted as a split-plot in a randomized complete block design with three replications. The main factor was different seasons of the year in four levels and the second factor was the substrates in four levels. The effect of different substrates on thermo-dynamic factors of the substrates and on morphological, qualitative, and water use efficiency characteristics of the plants were significant at the $5 \%$ probability level. The results showed that the temperature and humidity of the substrates containing organic compounds were higher than those of the soil. Also, plant height, number of nodes, percentage of coverage, and water use efficiency of the plants were higher in the growing medium containing mushroom compost. Having a high water use efficiency and keeping its qualitative characteristics to an acceptable level in the summer and spring, Frankenia was found to be a suitable species in these seasons. However, due to the cold-induced deleterious effects in winter on these characteristics, it was not a suitable plant species for external green wall systems in climatic conditions of the winter season of Mashhad. Based on this study's results, we suggest adding washed mushroom compost to the usual green wall substrate. Frankenia can also be considered a suitable plant for external green walls in spring, summer, and autumn in similar weather conditions to Mashhad.
\end{abstract}

Keywords: Vertical system, Sustainable development, Ground cover plants, Water use efficiency, Recycled material

1.2 and 3. M.Sc. Student and Associate Professor, Respectively Department of Horticulture and Landscape and Professor, Department of Soil Science, Faculty of Agriculture, Ferdowsi University of Mashhad, Mashhad, Iran.

*: Corresponding Author, Email: fatemeh.kazemi@um.ac.ir 